MacDonald, Heather (1996): Why Business Improvement Districts Work? in: Civil Bulletin, 4 (May 1996), http://www.manhattan-institute.org/html/cb_4.htm [10.09.2007].

Mallet, Wiliam J. (1994): Managing the Post-industrial City: Business Improvement Districts in the United States, in: Area, 26/3, 276-287.

McArdle, Andrea; Tanya Erzen (Hg., 2001): Zero Tolerance. Quality of Life and the New Police Brutality in New York City, New York.

Mitchell, Jerry (1999): Business Improvement Districts and Innovative Service Delivery (Grant Report), New York.

Nagel, Udo (2005): Bettelverbot auf Mönckebergstraße, Jungfernstieg und Rathausmarkt. In: Hamburger Abendblatt, 30.12.2005.

Neuer Wall Hamburg (Hg., 2005): Antrag zur Einrichtung eines Innovationsbereichs, http://www.bid-neuerwall.de/cms/upload/pdf/antrag_bid_neuer_wall.pdf [22.09.2007].

Parenti, Christian (1999): Lockdown America. Police and Prisons in the Age of Crisis. New York.

Reeves, Alan (1998): The Panopticisation of Shopping. CCTV and Leisure Consumption. In Clive Norris, Gary Armstrong, Jade Moran (Hg.): Surveillance, Closed Circuit Television and Social Control. Aldershot, 69-87.

Rigakos, George S. (2007): Polizei konsumieren. Beobachtungen aus Kanada. In: V. Eick; J. Sam bale; E. Töpfer (Hg.): Kontrollierte Urbanität. Zur Neoliberalisierung städtischer Sicherheitspolitik, Bielefeld, $39-54$.

Roost, Frank (2000): Der neue Times Square. Null Toleranz, in: arch+, 152/153, 104-109.

Sambale, Jens (2003): Nonprofits in Los Angeles: Between Peace-keeping Functions and Em ployment Policies. In: Volker Eick; Margit Mayer; Jens Sambale (Hg.): From Welfare to Work. Nonprofits and the Workfare State in Berlin and Los Angeles. Berlin, 81-86.

Sambale, Jens (2007): Skid Row Los Angeles: Genese eines Devianz- und Kontrollraumes, In: V. Eick; J. Sambale; E. Töpfer (Hg.): Kontrollierte Urbanität. Zur Neoliberalisierung städtischer Sicherheitspolitik, Bielefeld, 165-192

Schneider, Christiane (2007): Große Koalition der Vertreibung, 04.09.2007. http://www.hier- ist-dielinke-hamburg.de/cms/website.php?id=/de/aktuelles/040907vertreibung.html\&sid= $90 \mathrm{e} 689 \mathrm{a} 0225 \mathrm{~d} 364 \mathrm{def} 8 \mathrm{e} 8 \mathrm{f} 2162888 \mathrm{~b} 20$ [15.09.2007].

Schreiber, Frank; Volker Eick (2005): BID - kommunalpolitische und abgabenrechtliche Anmerkungen zu einem neuen Instrument (Manuskript). Wiesbaden/Berlin.

Senat der Freien und Hansestadt Hamburg (Hg., 2004a): Gesetz zur Stärkung der Einzelhandelsund Dienstleistungszentren (28. Dezember 2004, 2130-10), Hamburg. http://hh.juris.de/hh/gesamt/ZentrStaerkG_HA.htm\#ZZntrStaerkG_HA_P1 [18.03.2007].

Senat der Freien und Hansestadt Hamburg (Hg., 2004b): BID-Gesetzentwurf - Begründung, Hamburg, http:/fhh.hamburg.de/stadt/Aktuell/behoerden/stadtentwicklung-umwelt/ stadtplanung/bid/start.html [28.08.2007].

Stokes, Richard (2002): Place management in commercial areas: Customer service representatives in Philadelphia's central business district. In: Security Journal, 15/2, 7-19.

Töpfer, Eric (2007): Entgrenzte Raumkontrolle? Videoüberwachung im Neoliberalismus. In: V. Eick; J. Sambale; E. Töpfer (Hg.): Kontrollierte Urbanität. Zur Neoliberalisierung städtischer Sicherheitspolitik, Bielefeld, 193-226.

Ward, Kevin (2007): Business Improvement Districts: Policy Origins, Mobile Policies and Urban Liveability. In: Geography Compass, 1/3, 657-672.

Wehrheim, Jan (2002): Die überwachte Stadt. Sicherheit, Segregation und Ausgrenzung, Opladen.

\section{Henrik Lebuhn}

\section{Sozialräumliche Konflikte in der unternehmerischen Stadt}

\section{Eine Berliner Fallstudie über städtische Bewegungspolitik unter den Bedingungen von New Public Management}

Der Begriff der 'unternehmerischen Stadt' gehört mittlerweile zum festen Bestandteil der kritischen Stadtforschung. Das Ende der 1980er Jahre von David Harvey geprägte Konzept beschreibt die radikale Redefintion des städtischen Raums nach ökonomischen Kriterien. (Harvey 1989) Unter den Bedingungen der Krise des Sozialstaates, zunehmender Kapitalmobilität und verschärfter Weltmarktkonkurrenz unterwerfen sich Städte immer stärker einer betriebswirtschaftlichen Logik. Sie werden zu Marktakteuren, die um Kapitalinvestitionen, Arbeitsplätze und Steuergelder konkurrieren (siehe etwa Clarke/Gaile 1998: 55-88; Jessop 1997; Mayer 1995). Stadtpolitik, so Neil Brenner und Susanne Heeg, wird seit Mitte der 1980er Jahre immer stärker von Themen der wirtschaftlichen Entwicklung, industriellen Wachstums und struktureller Wettbewerbsfähigkeit dominiert (Brenner/Heeg 1999: 104).

Dass Städte immer mehr wie Unternehmen geführt werden, betrifft nicht nur ihre 'externe' Ausrichtung auf Wettbewerb und Wachstum. Auch die 'interne' Reorganisation der lokalen Verwaltungen nach betriebswirtschaftlichen Effizienzkriterien stellt ein Kernstück der Transformation zur 'unternehmerischen Stadt' dar. Die Einführung von Gobalhaushalten, Kosten- und Leistungsrechnungen und Controlling-Maßnahmen auf der kommunalen Ebene erzeugt bei stadtpolitischen EntscheidungsträgerInnen und in den lokalen Ämtern und Behörden eine neue Handlungsrationalität und ökonomisiert die öffentlichen Institutionen in bislang ungekanntem Ausmaß. Weltweit werden diese Reformen des öffentlichen Sektors unter dem Label New Public Management implementiert. Im Folgenden werden Funktionsweise und Wirkung von New Public Management (NPM) dargestellt und sein Einfluss auf politische Mikrokonflikte um den öffentlichen Stadtraum diskutiert. Wenn unternehmerische Stadtpolitik samt ihren ausschließenden und polarisierenden Konsequenzen soziale Widerstände provoziert (Mayer 2000) - d.h. individuelle und subversive Alltagspraxen, progressive soziale Bewegungen, aber auch konservative Not-in-myBackyard-Mobilisierungen -, dann gilt es zu fragen, wie das politische Terrain beschaffen ist, auf dem diese sozialräumlichen Konflikte ausgetragen werden.

PROKLA. Zeitschrift für kritische Sozialwissenschaft, Heft 149, 37. Jg., 2007, Nr. 4, 529-545 
Vor diesem Hintergrund werde ich argumentieren, dass NPM-Techniken nicht einfach nur eine an Sparzwängen orientierte Verwaltungsmodernisierung darstellen. Vielmehr müssen sie als eine Machttechnik verstanden werden, durch welche gerade die außerparlamentarischen Widerstände gegen die in-WertSetzung des städtischen Raums strukturell geschwächt werden. Hinter scheinbar neutralen Begriffen wie 'Modernisierung', 'Dezentralisierung' und 'Entbürokratisierung' verbirgt sich der zuticfst politische Charakter von NPMTechniken. Durch sie werden wirkmächtige ökonomische Handlungsrationalitäten implementiert. Zentrale Fragen lokaler Politik werden der öffentlichen Aushandlung dadurch tendenziell entzogen und statt dessen in bürokratische Entscheidungsverfahren überführt.

Im folgenden stelle ich zunächst die Grundprinzipien des NPM dar. Im Anschluss illustriere ich ihre Wirk- und Funktionsweise am Beispiel Berlins, wo die kommunalen Verwaltungen seit 1996 massiv (konter-)reformiert wurden. Der Konflikt um ein Soziales Zentrum, der von 2001 bis 2005 zwischen auBerparlamentarischen Basisgruppen aus dem Umfeld des Berliner Sozialforums einerseits und VertreterInnen des Bezirksamtes Friedrichshain-Kreuzberg und der Berliner Landesregierung andererseits ausgetragen wurde, dient als Fallbeispiel, um die Konsequenzen von NPM-Reformer auf sozialräumliche Mikrokonflikte aufzuzeigen.

\section{New Public Management}

Der Begriff 'New Public Management' bezeichnet, um es auf eine griffige Formel zu bringen, die Neuausrichtung von Verwaltungstätigkeiten gemäß betriebswirtschaftlicher Effizienzkriterien. Als Reaktion auf die Finanzierungskrise des Wohlfahrtsstaates wurde das Konzept des NPM ab Mitte der 1980er Jahre weltweit entwickelt und implementiert; Vorreiterrollen hatten die Niederlande, Großbritannien, die USA, Australien, Kanada, Neuseeland und Skandinavien. Seitdem spielen NPM-Reformen eine zentrale Rolle beim neoliberalen Ab- und Umbau sozialstaatlicher Funktionen und bei der Privatisierung offfentlicher Räume und Güter. Während die Verschiebung der Prioritäten bei der Investition öffentlicher Mittel hin zu wettbewerbs- und standortpolitischen Maßnahmen eine Ökonomisierung von Stadtpolitik auf der PolicyEbene darstellt - also unternehmerische Stadtpolitik im engeren Sinne - zielen NPM-Reformen auf eine Ökonomisierung der öffentlichen Institutionen selbst abs wirken also auf der Polity-Ebene. Was die konkrete Funktionsweise der Reformen angeht, lassen sich generell fünf Bereiche unterscheiden (einen guten Überblick gibt Jäggi 1996):

1 Die hier zitierten Interviews habe ich im Rahmen der Feldforschung für meine Dissertation über stadträumliche Privatisierungskonflikte in Berlin und Los Angeles erhoben (Lebuhn 2008).

2 In der deutschsprachigen Debatte wird oft auch von, Neuen Steuerungsmodellen' gesprochen.
- Wirkungsorientierung: Die politischen EntscheidungsträgerInnen steuern die Tätigkeit der Verwaltung nicht mehr mittels detaillierter Vorgaben hinsichtlich der finanziellen und personellen Ressourcen (Input), sondern durch die Vorgabe der zu crreichenden Leistungen (Output) und der gewünschten Wirkungen (Outcome).

- Globalbudgets: Die zuständige politische Instanz stellt ein Gesamtbudget zur Verfügung, mit dem die Verwaltung oder einzelne Verwaltungsabteilungen die vorgegebenen Aufgaben so effizient wie möglich erfüllen müssen. Im Unterschied zur traditionellen Budgetierung enthält das Globalbudget keine zweckgebundene Zuweisung von Einzelposten sondern eine Kontraktsumme, über welche die Verwaltung selbstständig verfügen kann.

- Leistungsmessung: Die Leistungen der Verwaltung wird nach sogenannten 'Produkten' definiert und die Ergebnisse in monetären GröBen messbar gemacht. Mit der Definition und Bereitstellung von Produkten wird den NutzerInnen öffentlicher Leistungen darüber hinaus ein Status als KundInnen zugewiesen, die die betreffenden Produkte 'konsumieren'.

- Wettbewerb: Qualität und Effizienz der Tätigkeit soll außerdem durch die Einführung von Marktelementen, also durch den Leistungsvergleich zwischen verschiedenen Verwaltungseinheiten und/oder Privatunternehmen erreicht werden. Alternativ dazu können auch Benchmarks formuliert werden.

- Controlling: Interne und/oder externe Gremien oder Unternehmen überprüfen regelmäßig die Leistungen der Verwaltung und ihre Wirkungen nach betriebswirtschaftlichen Kriterien und schlagen, wenn nötig, Verbesserungen vor.

Die Ziele der NPM-Reformen sind also eine Rationalisierung und Ökonomisierung öffentlicher Tätigkeiten, Beschränkung des öffentlichen Sektors auf Kernkompetenzen, Einsparung der Mittel für öffentliche Aufgaben, Personalkürzung, etc. Die Stadt wird 'intern' nach dem Vorbild eines privaten Unternehmens organisiert. Zwar dürfte es sich mittlerweile auch unter den VertreterInnen des NPM-Ansatzes herumgesprochen haben, dass privatwirtschaftliche Modelle nicht unmittelbar auf den öffentlichen Sektor übertragbar sind - und zwar unabhängig davon, ob dies überhaupt wünschenswert ist oder nicht. ${ }^{3}$ Darüber hinaus scheinen NPM-Reformen selten die versprochenen Ergebnisse von Haushaltskonsolidierung und Verbesserung der öffentlichen Dienste zu

3 Siehe etwa die kritischen Ergebnisse des umfangreichen Forschungsprojekts „Zehn Jahre Neues Steuerungsmodell: Evaluation lokaler Verwaltungsmodernisierung", das an der Universität Konstanz in Kooperation mit den Universitäten HU Berlin, Potsdam und Marburg durchgefuihrt wurde (Bogumil, et al. 2006; Kublmann 2006). 
erbringen. Doch tut dies der Popularität des Ansatzes keinen Abbruch. Kein Wunder, denn NPM-Techniken sind, wie wir sehen werden, viel mehr als nur der Versuch einer fiskalpolitischen Krisenlösung.

\section{Verwaltungsreformen in Berlin}

In Berlin setzten die Vorbereitungen zur Einführung von NPM-Techniken Mitte der 1980er Jahre ein. ${ }^{4}$ „Ausgehend von den Ergebnissen einer EnqueteKommission zur Neuordnung der Finanzstruktur im Land Berlin aus dem Jahr 1984 wurde vom Land Berlin ein Gutachten in Auftrag gegeben, mit dem entsprechende Modelle zur Neuordnung erarbeitet werden sollten." (Fischer 1999: 3f.). Neben der Einführung einer Kosten- und Leistungsrechnung (KLR) wurden die Budgetierung und das betriebswirtschaftliche Controlling der Haushalte der Senats- und der Bezirksverwaltungen vorgeschlagen - also alle zentralen Elemente des NPM-Ansatzes. „Hauptziele im Gesamtprojekt sind eine Erhöhung der Wirtschaftlichkeit des Verwaltungshandelns und eine Schärfung des Kostenbewußtseins," so die Senatsverwaltung Berlin in einer Reformkonzeption aus dem Jahr 1995 (Senatsverwaltung 1995: 3).

Nach einer ersten Phase der Erarbeitung konkreter Modelle, begann die Umstrukturierung der öffentlichen Verwaltungen ab 1994/1995 unter dem Etikett der 'Verwaltungsmodernisierung'. Die Berliner Verwaltungen stellten erstmals systematisch Betriebswirte ein. Diese sollten die Implementierung der KLR und die betriebswirtschaftliche Controlling-Funktion in den Verwaltungen übernehmen. Auf Grundlage der KLR wurde dann die bis dahin übliche kameralistische Finanzmittelzuweisung - das heißt die zweckgebundene Zuweisung der Haushaltsmittel auf der Grundlage von Erfahrungswerten - sukzessive durch die Zuweisung einer Globalsumme ersetzt. Eine zentrale Rolle spielte dabei die Definition von etwa 1500 'Produkten', die eine 'output-' und 'outcomeorientierte' Steuerung der Verwaltung ermöglichen soll (Senatsverwaltung 1995: 7). Dr. Margrit Köhler, Leiterin des Steuerungsdienstes im Bezirksamt Friedrichshain-Kreuzberg, erklärt im Interview die Funktionsweise der KLR und der Budgetbemessung auf Ebene der Bezirksverwaltungen:

„1994/1995 wurde damit angefangen, dass für die Bezirke Produkte definiert wurden. (...) Ein Produkt ist zum Beispiel, was ich an kulturellen Leistungen im Bezirk anbiete. Kulturberatung zum Beispiel. (...) Da muss ja jetzt entschieden werden, wieviel Geld stecken wir in dieses Prozum Beispiel. (...) Da muss ja jetzt entschieden werden, wieviel Geld stecken wir in dieses Pro-
dukt und mit Hilfe der Kostenrechnung werden jetzt die Kosten für dieses Produkt erfasst. (...) Wenn ich für ein Produkt arbeite, dann erfasse ich die Zeit, die ich dafür aufbringe. Und zwar prozentual. Also ich habe heute einen halben Tag für das Produkt xy gearbeitet. Dann wird das notiert. Und das erfolgt sozusagen über einen Monat, dass jede Kraft eigentlich notiert, für welche Produkte sie innerhalb eines Monats gearbeitet hat. Und am Monatsende steht dann fest: 10 Prozent fïr dieses Produkt 15 Prozent fiir ienes. Und dann gibt es auch noch sogenannte NPT das ist eine nicht-produktbezogene Tätigkeit. (...) Oder wenn ein Mitarbeiter nur ein einziges

4 Einen guten Überblick über die Implementierung und die Funktionsweise der NPMReformen in Berlin bieten: Senatsverwaltung Berlin (1995), (2004)
Produkt bearbeitet, dann kommt die gesamte Zeit, auch die nicht-produktbezogene Tätigkeit, (...) die Krankheitszeit und die Urlaubszeit kommt auf das Produkt. Das Produkt muss das mit tragen. Weil ja wie in einem Unternehmen... da müssen ja auch alle Kosten auf die Produkte der Unternehmen kommen, und über den Preis auf dem Markt wird dann das Geld wieder hereingeholt. Und so ist das bei uns auch. ${ }^{65}$

In regelmäßigen zeitlichen Abständen legen alle Bezirke ihre Kosten- und Leistungsrechnungen dem Land vor. Nun kann die wirtschaftliche Effizienz der Bezirke im direkten Vergleich abgelesen werden: Für jedes Produkt (und jeden Bezirk) wird dargestellt, wie hoch die realen Kosten waren, um das Produkt über einen bestimmten Zeitraum für jeweils 1000 EinwohnerInnen bereit zu stellen.

Zur Errechnung der vom Land an die Bezirke vergebenen Haushalte wird für jedes Produkt ein Ranking erstellt: Der Bezirk, der seinen 'KundInnen' - also den Bürgern und Bürgerinnen, die die betreffende öffentliche Leistung in Anspruch nehmen - das Produkt am günstigsten anbieten konnte, nimmt den obersten Rang ein; der Bezirk, der am teuersten produziert hat, den letzten. Nun werden die mittleren beiden Ränge addiert, bei zwölf Bezirken in Berlin also der sechste und siebte Rang, und durch zwei geteilt. Die so errechnete Summe wird als 'Median' bezeichnet. Der Median gilt als Richtwert für die 'angemessenen' Kosten zur Bereitstellung des jeweiligen Produkts und stellt die Grundlage für die Errechnung und Zuweisung der Globalhaushalte an die Bezirke dar. Über ihre Haushalte wiederum können die Bezirke dann selbstständig verfügen. Cornelia Reinauer, Bezirksbürgermeisterin von Friedrichshain-Kreuzberg (PDS), erklärt im Interview:

„Die Bezirke haben ja jetzt tatsächlich die Kosten- und Leistungsrechnung. Also das heißt seit zehn Jahre bilden wir jetzt ab: Was kosten zum Beispiel unsere Gebäude in der Unterhaltung. Und wir kriegen ja jetzt Gelder danach zugewiesen. Wie teuer sind wir mit unserem Personal, und und und... da ist ein Riesendruck entwickelt worden. (...) Wir können jetzt tatsächlich mit einem unheimlichen bürokratischen Aufwand über zehn Jahre sagen, was das jetzt in unterschiedlichen Bezirken kostet. Und dann gibt es einen sogenannten Median. Und wenn Sie da drunter oder drüber liegen, dann ist klar: Wenn Sie drunter liegen, dann wirtschaften Sie gut. Dann gehen Sie mit ihren Ressourcen gut um. Und wenn Sie drüber liegen, dann müssen Sie gucken, woran liegt's? Liegt's am Personal, liegt's an den Gebäuden?"“6

Die KLR soll jedoch nicht nur eine in monetären Größen ausgedrückte Vergleichbarkeit der Leistungen der Bezirke untereinander ermöglichen, sondern wirkt auch als das zentrale Instrument für die finanzielle Maßregelung der Bezirke durch das Land Berlin: Hat ein Bezirk in einer Produktgruppe 'unnötige' Verluste erwirtschaftet, dann werden diese Kosten 'zur Strafe' von seinem Budget abgezogen. Dies betrifft zum Beispiel kalkulatorische Zinsen und Unterhaltungskosten für nicht genutzte öffentliche Grundstücke und Gebäude,

5 Projektinterview mit Dr. Margrit Köhler, Leiterin des Steuerungsdienstes im Bezirksamt Friedrichshain-Kreuzberg, 8. Olctober 2004.

6 Projektinterview mit Cornelia Reinauer (PDS), Bezirksbürgermeisterin FriedrichshainKreuzberg, 22. September 2004. 
sogenannte 'budgetunwirksame Kosten'. Im Fall der kalkulatorischen Zinsen für ein Grundstück etwa wird veranschlagt, dass das im Grundstück festgelegte Vermögen potentiell einen bestimmten Zins erwirtschaften könnte? Wenn das Gelände ungenutzt bleibt, geht diese (potentielle) Einnahme verloren. Die betreffende Summe geht als Kostenpunkt in die KLR ein und wird bei der Budgetvergabe vom errechneten Haushalt abgezogen. Der Bezirk wird also für sein 'ineffizientes Wirtschaften' durch Mittelkürzung bestraft. Als Ausweg bleibt die schnelle Veräußerung ungenutzter öffentlicher Räume über den Liegenschaftsfonds. ${ }^{8}$ Dr. Margit Köhler erklärt im Interview:

„Das ist der Ausgangspunkt für die Budgetierung, für die Zuweisung. (...) Wenn ich sehr viele Gebäude habe, habe ich auch sehr viele budgetunwirksame Kosten. Und dadurch werde ich gedrückt, mich auf möglichst engem Raum zu bewegen. (...) Der Bezirk wird damit praktisch gezwungen, dass er überflüssige Gebäude abgibt. Ansonsten habe ich Budgetverluste. Das ist also eine ganz wichtige Position."

Hier wird deutlich, dass die neuen NPM-Techniken eine starke fiskalpolitische Handlungsrationalität erzeugen, durch die ungenutzte öffentliche Liegenschaften einem unmittelbaren Privatisierungsdruck unterworfen werden. Und das hat, wie ich am Berliner Beispiel zeigen werde, weitreichende Folgen für die sozialräumlichen Konflikte, die in der unternehmerischen Stadt zwischen privatwirtschaftlichen Akteuren, VertreterInnen des lokalen Staates und städtischen sozialen Bewegungen ausgetragen werden.

\section{Der Konflikt um ein Soziales Zentrum in Berlin}

Mit der Besetzung eines leer stehenden Gebäudes im Besitz der Dienstleistungsgewerkschaft Verdi begann im November 2001 der vierjährige Konflikt um ein Soziales Zentrum in Berlin. In hohem Maße, so meine These, wurde dieser durch die oben dargestellten NPM-Reformen der Berliner Verwaltungen strukturiert - und zwar zu Ungunsten der außerparlamentarischen Forderung nach einem Sozialen Zentrum.

Im Mittelpunkt des Konflikts stand auf der einen Seite die „Initiative für ein Soziales Zentrum“, die sich ab 2001 im Umfeld des Berliner Sozialforums ${ }^{10}$ gründete und organisierte (zum Berliner Sozialforum siehe Bahn/Haberlan 2004; felS 2004). Mit ihrem Netzwerk- und Kampagnencharakter brachte sie (post-)autonome Gruppen, ehemalige HausbesetzerInnen, Einzelpersonen und Initiativen aus dem Spektrum der globalisierungskritischen Bewegung sowie -

7 Der Begriff kalkulatorische Zinsen entstammt der Betriebswirtschaftslehre, bzw. dem Rechnungswesen. Vereinfacht gesagt handelt es sich dabei um Zinsen, die erzielt worden wären, wenn Kapital - statt es im Unternehmen zu investieren - auf dem Kapitalmarkt angelegt worden wäre.

8 Siehe http://www.liegenschaftsfonds-berlin.de/ (Zugriff am 25. Juli 2005)

9 Projektinterview mit Dr. Margrit Köhler, Leiterin des Steuerungsdienstes im Bezirksamt Friedrichshain-Kreuzberg 8. Oktober 2004 .

10 Siche http://www.sozialforum-berlin.de (Zugriff am 30.4. 2007) zumindest dem Anspruch nach - auch migrantische und Erwerbsloseninitiativen zusammen. Um der inhaltlichen Annäherung und Kooperation dieser Gruppen eine räumliche Infrastruktur und Verankerung zu geben, forderte die Initiative von der Stadt Berlin einen öffentlichen Raum für Stadtteilarbeit, soziale Projekte und Basisgruppen. " Dabei stellte die Initiative von Anfang an klar, dass sie nur einen 'politischen Preis' (die Betriebskosten) zu zahlen bereit war, nicht aber den Marktpreis einer öffentlichen Liegenschaft.

Da die Initiative vor allem im Stadtteil Friedrichshain-Kreuzberg organisiert war und hier auch Aussichten auf die Nutzung leer stehender öffentlicher Räume zu bestehen schienen, richtete sich ihre Forderung an VertreterInnen des Bezirksamtes Friedrichshain-Kreuzberg bzw. an VertreterInnen des Landes Berlin, insoweit diese politische Ebene mit betroffen war. Die zentrale Protagonistin auf der Seite der Stadt war dem entsprechend die zuständige Bezirksbürgermeisterin Cornelia Reinauer (PDS): Gemeinsam mit ihren Stadträten verhandelte sie mit der Initiative, musste die unterschiedlichen Einschätzungen der politischen und wirtschaftlichen Situation vor Ort abwägen, konfligierende Interessen austarieren, stand im öffentlichen Rampenlicht und hatte den Ausgang des Konflikts politisch zu verantworten.

Angesichts der Tatsache, dass zu Beginn der Kampagne im Liegenschaftsfonds der Stadt Berlin mehrere tausend ungenutzte Immobilien auf ihre Privatisicrung warteten, schien die Forderung der Initiative trotz der Spar- und Kürzungspolitik zunächst nicht völlig unrealistisch. Geeignete Häuser für ein Soziales Zentrum gab es mehr als genug - das war keine Frage. Doch die Bezirksbürgermeisterin Cornelia Reinauer und die zuständigen PolitikerInnen auf Kommunalebene und auf Landesebene sahen das anders. Ebenso die Berliner Polizei, die im Laufe des Konflikts mehrfach intervenierte und den öffentlichen Leerstand gegen politische Besetzungen der Initiative verteidigte. Zwar konnte die Initiative für ein Soziales Zentrum nach der Besetzung des VerdiGebäudes im Herbst 2001 noch mit zwei weiteren spektakulären Besetzungen leer stehender öffentlicher Gebäude auf sich aufmerksam machen: am 9. Oktober $2003 \mathrm{im}$ Stadtteil Kreuzberg ${ }^{12}$, sowie am 3. April 2004 in der Oranienburger Straße im Stadtteil Berlin-Mitte. ${ }^{13}$ Im Herbst 2003 konnte die Initiative gar eine mehrmonatige Verhandlung mit VertreterInnen der Bezirksregierung ${ }^{14}$

11 Indymedia (2002): Soziales Zentrum Berlin, Nachruf, 7. Juni 2002; http://www.stressfaktor. squat.net/2002/berlin_10.html (Zugriff am 28.11.2006).

12 Indymedia (2003): Besetzung für ein Soziales Zentrum Berlin, 9.10. 2003, http://germany.indymedia.org/2003/10/63139.shtml (Zugriff am 28.11. 2006); sowie taz, 13. Oktober 2003, S. 21 und Neues Deutschland, 11. Oktober 2003.

13 Online-Bericht des RBB; http://www.rbb-online.de/_/nachrichten/politik/beitrag_jsp/ key=news317772.html (Zugriff am 5.9. 2004)

14 Der Begriff, Bezirksregierung' fasst hier mehrere Organe zusammen: die Bezirksverordnetenversammlung (BVV) ist vom Bezirksamt (BA) zu unterscheiden. Letzteres setzte sich aus der Bezirksbürgermeisterin und fünf Stadträtinnen und Stadträten zusammen. Darüber hinaus 
Friedrichshain-Kreuzberg erwirken. ${ }^{15}$ Doch am Ende zeigten sich die verantwortlichén LokalpolitikerInnen kompromisslos. Immer wieder betonte die Bezirksbürgermeisterin Cornelia Reinauer, dass ihr vor allem wegen der angespannten Finanzsituation des Landes Berlin die Hände gebunden seien. So sehr sie auch wolle - die öffentlichen Räume des Bezirks seien einfach nicht 'zum Nulltarif zu haben. Leer stehende Immobilien dürften, so die Vorgabe des Berliner Senats, nur zu Marktpreisen vermietet werden und würden andernfalls durch den Berliner Liegenschaftsfonds privatisiert. Zusätzlich zu dem Konflikt über die Finanzierung des Sozialen Zentrums schien es vor allem seitens des Berliner Innensenators Ehrhart Körting (SPD) auch politische Vorbehalte gegenüber den linksradikalen Gruppen im Umfeld der Initiative zu geben, was die Verhandlungen zusätzlich erschwerte (taz-Interview, 13. 10. 2003, S. 21). Nachdem im Herbst 2004 die mehrmonatige Verhandlung um eine leer stehende Kindertagesstätte im Stadtteil Kreuzberg ohne Ergebnis endete (Berliner Zeitung, 3. 9. 2004, S. 28), verlief der Konflikt im Laufe des Jahres 2005 mehr oder weniger im Sande. Viele an der Initiative beteiligten Gruppen fanden individuelle (Not-)Lösungen für ihren Raumbedarf. Mit der Besetzung des 'Haus Bethanien' (Kreuzberg) durch das polizeilich geräumte Wohn- und Stadtteilprojekt in der Yorkstraße (Kreuzberg/Schöneberg, http://www.yorck59.net), sowie durch das studentische Projekt einer 'Offenen Uni' an der HumboldtUniversität (http://www.offeneuni.tk) wurde die Idee eines großen Sozialen Zentrums unabhängig von der Initiative zumindest in Teilen realisiert. Doch das zentrale Anliegen der Initiative für ein Soziales Zentrum - die räumliche und infrastrukturelle Zusammenführung einer Vielzahl jüngerer linker Basisgruppen in einer öffentlichen Liegenschaft - wurde nicht verwirklicht. Im Folgenden diskutiere ich zwei zentrale Wirkweisen bzw. Konsequenzen der Berliner NPM-Reformen, durch die der Konflikt um das Soziale Zentrum erheblich strukturiert wurde.

\section{Privatisierungsdruck durch New Public Management}

Im Konflikt um das Soziale Zentrum in Berlin äußerten viele AktivistInnen, dass das finanzpolitische Argument der LokalpolitikerInnen, es gäbe kein Geld für ein Soziales Zentrum, vorgeschoben sei. Als 'Beweis' dafür wurde angeführt, dass Berlin ja ständig Gelder in städtische Projekte investiere. Dieses Argument ist nicht falsch, aber genauso wenig ist es richtig. Denn unternehmerische Stadtpolitik heißt gerade nicht, dass es überhaupt keine öffentlichen Mit-

verfïgt der Bezirk über eine eigene Verwaltung. Auf der Landesebene waren vor allem der Innensenator Ehrhart Körting (SPD), sowie die Senatsverwaltung für Stadtentwicklung von dem Konflikt betroffen.

15 taz, 3. November 2003, S. 21; Der Tagesspiegel, 13. Dezember 2003; ausführlicher: Sozialforum Berlin (2004): Chronologische Übersicht zu den Verhandlungsschritten - Juli 2003 bis Januar 2004; http://www.sozialforum-berlin.de (Zugriff: 14.10. 2004) tel mehr gäbe. Doch der fiskalpolitische Druck bewirkt, dass die vorhandenen Gelder unter der Maßgabe der Rentabilität in spezifische Projekte kanalisiert werden. Öffentliche Ausgaben werden darauf befragt, ob sie im Hinblick auf die Herstellung eines günstigen Investitionsklimas und die Schaffung öffentlicher Revenuen 'sinnvoll' sind, ob 'sie sich lohnen'. NPM-Techniken spielen eine wichtige Rolle dabei, diese Handlungsrationalität bis auf die Ebene kommunaler Mikropolitik durchzusetzen.

So erhielt der Berliner Bezirk Friedrichshain-Kreuzberg mit der Einführung des Globalhaushaltes zunächst einmal ein hohes Maß an Autonomie. Die PolitikerInnen und VerwaltungsmitarbeiterInnen des Bezirks können nun selbstständig entscheiden, wieviel Mittel für welche Maßnahmen aufgewendet werden sollen. Doch agieren sie dabei unter den Bedingungen einer permanenten Mittelverknappung, „Die Gesamtsumme für die Bezirke wird von Jahr zu Jahr gekürzt. Sie wissen ja wie die finanzielle Situation des Landes Berlin ist ${ }^{\text {"6 }}$ wie Dr. Margit Köhler im Interview feststellt. Anstatt jedoch gegen den rigiden Sparkurs des Senats zu protestieren, macht sich der Bezirk die Sparpolitik zu eigen. Denn Autonomie in der Haushaltsplanung heißt auch, mit dem 'eigenen Budget' effizient und sparsam zu wirtschaften. In der Wahrnehmung des Bezirks kommen die Sparauflagen zwar 'von oben', müssen aber auf Grund der autonomen Haushaltsführung selbstverantwortlich konkretisiert und durchgeführt werden. Die Verknappung der Haushalte auf Landesebene gibt sich dabei den Schein von wissenschaftlicher Neutralität und logischer Stringenz. Denn die Höhe der Haushalte wird über die Feststellung eines Medians mathematisch errechnet. Mit diesem Verfahren wird zugleich sichergestellt, dass die Globalhaushalte der Bezirke kontinuierlich verknappt werden - eine Dilemma-Situation, die sich die MitarbeiterInnen in den Bezirksregierungen und kommunalen Verwaltungen offenbar überhaupt nicht bewusst machen: Haushaltet ein Bezirk schlecht und liegt mit seinen Ausgaben über dem errechneten Median, so werden ihm budgetunwirksame Kosten vom Haushalt abgezogen. Hat er dagegen 'gut gewirtschaftet' und bleibt bei der Bereitstellung der 'Produkte' unter dem Median, trägt der Bezirk dazu bei, dass der errechnete Mittelwert zur Finanzierung der Produkte und der Bezirk sinkt und in der Folge die Haushalte aller Bezirke verknappt werden.

Die mit einem unglaublichen bürokratischen Aufwand durchgeführte akribische Erfassung der Arbeitszeiten und Personalkosten, die zur Bereitstellung der einzelnen Produkte aufgewendet werden, erzeugt bei den politischen EntscheidungsträgerInnen und bei den VerwaltungsmitarbeiterInnen einen grundlegenden Bewusstseinswandel. Die an monetären Größen gemessene Rationalität und Effizienz von Verwaltungshandeln wird in das Alltagsbewusstsein der MitarbeiterInnen eingelassen. Dr. Margit Köhler, die in FriedrichshainKreuzberg die Implementierung der KLR verantwortlich geleitet hat, berichtet im Interview, dass es 
,schon eine Riesenumstellung (war), als wir mit der Kostenrechnung angefangen haben und dann jeder die Arbeitszeit erfassen und aufschreiben musste. Und dann auch alle Kosten, die anfallen, nach Möglichkeit Produkten zuzuordnen. Das hat schon ein großes Umdenken und erstmal auch große Unruhe verursacht. Inzwischen ist das völlig normal. (...) Es haben eben wirklich auch Veränderungen in den Denkprozessen und im ganzen Bewusstsein stattgefunden."

Bei Michel Foucault wird genau dieser Effekt im Rahmen seiner Studien zur 'Gouvernementalität' als eine Einlassung von Kontroll- und Herrschaftsmechanismen in die Subjekte selbst beschrieben (siche: Bröckling, et al. 2000; Foucault 2000; Foucault 1994). Gerade weil die Kontrolle nicht von außen, sondern als Selbstkontrolle durch die MitarbeiterInnen eingerichtet ist, wird die Unterwerfung der eigenen Tätigkeit unter betriebswirtschaftliche Kriterien nicht mehr als Fremdbestimmung, sondern als eigenverantwortliches und quasi-natürliches Handeln wahrgenommen.

Der fiskalpolitische Druck hat vor allem für nicht-kommerzielle Projekte erhebliche Konsequenzen. Denn was sich 'nicht rechnet' wird tendenziell ausgeschlossen oder bei der Vergabe öffentlicher Räume zumindest benachteiligt. Dies ist nicht etwa Gegenstand öfentlicher und politischer Aushandlung, sondern ergibt sich logisch aus den durch die NPM-Techniken erzeugten finanzpolitischen Handlungsrationalitäten. Wenn etwa der Unterhalt und die kalkulatorischen Zinsen für ungenutzte öffentliche Gebäude dem Bezirk vom Haushalt abgezogen werden und dieser in der Folge sein Raumkontingent auf ein Minimum verknappt, stehen schon allein deswegen zahlenmäßig weniger öffentliche Räume zur Verfügung. Für die noch vorhandenen öffentlichen Liegenschaften wird der Zugang eng an betriebswirtschaftliche Kriterien geknüpft: Kann der Nutzer oder die Nutzerin die Unterhaltskosten aufbringen? Ist er oder sie finanziell in der Lage, auch den vom Bezirk zu erwirtschaftenden Mietzins aufzubringen?

So setzen sich über scheinbar neutrale ökonomische Kriterien, die zunächst einmal alle potentiellen NutzerInnen betreffen, indirekt auch politische Präferenzen durch, die gerade linke und kritische Stadtteilprojekte strukturell benachteiligen. Denn 'Marktfähigkeit' ist in der Regel mit einem Minimum an politischem Konformismus verbunden. Projekte, die nicht-kommerziell operieren oder sich der kapitalistischen Verwertungslogik gar ausdrücklich entzichen wollen, 'verkaufen sich' eben weniger gut - oder gar nicht - und stehen bei einer an NPM-Kriterien gebundene Nutzung öffentlicher Ressourcen dann entsprechend schlecht da.

Im Fall des Konflikts um ein Soziales Zentrum in Berlin konnte sich die Bezirkverwaltung Friedrichshain-Kreuzberg auf der Grundlage der mit den NPMReformen implementierten Kriterien und Verfahren gegen die Vergabe öfentlicher Räume an Stadtteilgruppen und Basisinitiativen entscheiden, ohne dies

16 Projektinterview mit Dr. Margrit Köhler, Leiterin des Steuerungsdienstes im Bezirksamt Friedrichshain-Kreuzberg, 8. Oktober 2004. explizit politisch begründen zu müssen. Streng genommen ergab sich die Entscheidung gegen ein Soziales Zentrum automatisch bei der Befolgung der durch die NPM-Techniken vermittelten Logik. Polemisch gesagt, lässt sich die Entscheidung über die Vergabe eines öffentlichen Raums am Median errechnen. Dass die ökonomische Entscheidung in der Tat eine zutiefst politische Entscheidung ist, mag vielen Beteiligten vielleicht gar nicht bewusst sein. NPM ist also nicht so sehr eine Verwaltungsform oder Sparmethode, sondern vielmehr eine Machttechnik. NPM hat darüber hinaus einen hoch ideologischen Charakter, denn es spiegelt Neutralität, Sachzwänge und wissenschaftliche Stringenz vor, wo tatsächlich zutiefst politische und kontingente Entscheidungsprozesse in Interessen- und Klassenkonflikten ablaufen.

\section{Politische Konkurrenzen in der unternehmerischen Stadt}

Die Initiative für ein Soziales Zentrum setzte sich überwiegend aus Basisgruppen zusammen, sich auf einer lokalen Ebene außerparlamentarisch bzw. auBerhalb formaler Politikstrukturen engagieren, dabei in der Wahl ihrer Arbeitsbereiche jedoch politische Themen von überregionaler Bedeutung bearbeiten: Sozialpolitik, Kulturpolitik, Privatisierung öffentlicher Güter, Bildungspolitik, Rassismus und Fremdenfeindlichkeit, u.a.m. Nachdem die Initiative 2001 zunächst vor allem von Gruppen und Personen aus dem Spektrum der globalisierungskritischen Bewegung initiiert worden war, schlossen sich ihr später auch Gruppen wie 'Anders Arbeiten' und die 'Piqueteros' (benannt nach den argentinischen ArbeiterInnenprotesten) an. Im Zuge des aktuellen Umbaus des Sozialstaats und insbesondere der Hartz IV-Proteste schien es eine gegenseitige Annäherung zu geben: Viele sozialpolitisch engagierte Gruppen, die sich ab 2003 gegen die Politik der Schröder-Regierung formierten, hatten offensichtlich wenig Berührungsängste mit einer radikalen Gesellschaftskritik. Umgekehrt orientierten sich auch linksradikale und autonome Gruppen verstärkt an den jüngsten Sozialprotesten. Aktionsgruppen wie 'die Überflüssigen' (http://www.ueberfluessig.tk) oder 'Berlin Umsonst' (http://www.berlinumsonst.tk), die im Umfeld des Sozialforums und der Initiative für ein Soziales Zentrum entstanden und bundesweit für Aufmerksamkeit und Nachahmung sorgten, traten einerseits mit autonomen Politikformen wie Besetzungen und öffentlichkeitswirksamen Störaktionen in Erscheinung, agierten dabei aber andererseits auf 'klassisch' sozialpolitischen Politikfeldern.

Neben der unmittelbaren Durchsetzung des Sozialen Zentrums - und der räumlichen und infrastrukturellen Verankerung der beteiligten Gruppen - verfolgte die Initiative mit der Forderung nach einem Sozialen Zentrum noch ein zweites Ziel: die Politisierung und Mobilisierung anderer lokaler Gruppen gegen die Kürzungs- und Privatisierungspolitik des Berliner Senats. Der neoliberale Privatisierungskonsens in den Berliner Bezirken und auf Landesebene legte 
eine außerparlamentarische Strategie nahe, die auch Mittel des zivilen Ungehorsams und kontrollierten Regelbruchs einschloss. Eine 'Politik der ersten Person' sollte andere Gruppen und Initiativen ermutigen, die eigenen Bedürfnisse als legitime politische Forderungen in die Öffentlichkeit zu tragen. Wenn viele von der Berliner Spar- und Kürzungspolitik betroffene Akteure dem Beispiel der Initiative folgen und öffentliche Mittel und Räume 'umsonst' oder zu einem 'politischen Preis' für sich reklamieren, dann könnte dies einen „Aufstand des Gebrauchswerts gegen den Tauschwert" (Agnoli 1995: 55) in Gang setzen, so die Hoffnung. ${ }^{17}$ Bernhard Feder, Mitglied der Initiative, im Interview:

Denn „wenn jetzt einfach welche daherkommen und ein Haus bekommen, dann besteht natürlich die Gefahr - und von uns ist das ja auch sehr wohl gewollt - dass diese sozialen Projekte beim Senat auflaufen und sagen: Wieso wir nicht auch?! Wir wollen auch Räumlichkeiten!' Und dann haben sie (der Bezirk und der Senat; H.L.) ein großes Problem." "*

Seitens des Bezirks und des Landes wurde die Initiative für ein Soziales Zentrum dem entsprechend von Beginn an nicht nur als haushaltspolitisches Problem sondern auch als (sozial-)politische Bedrohung wahrgenommen. Dafür spricht nicht zuletzt, dass die Initiative auch dann noch 'leer ausging' als der Berliner Senat im November 2004 - zumindest formal - die Möglichkeit einer eingeschränkten Zwischennutzung leer stehender Gebäude eröffnete. ${ }^{19}$ Die PDS-Politikerin Cornelia Reinauer reagierte geradezu allergisch auf die widerständischen Strategien der Initiative, die sich nicht auf Verhandlungen am Runden Tisch beschränkten:

„Die haben also einfach widerrechtlich ein Haus in Besitz genommen. Und das ist ja auch wirklich schwierig. Nicht umsonst haben wir ja auch ordnungspolitische Regeln. Also wenn dann jetzt das jeder so macht. Das glauben sie jawohl nicht, wie andere Träger und Vereine darauf geachtet haben, wie wir diesen Konflikt lösen. Die auch alle bei uns stehen und sagen: (...) 'Und wir? Wir müsen jetzt auch nur mal ein bisschen strafrechtlich was machen und Euch unter Druck setzen, und dann kiegen wir shon alle?' Und das bo. Druck sezen, und dan ko ich wir shon alle?" Und zalforum auch gesagt. Wo ich mich dann auch geärgert habe. Wo ich gesagt habe: Liebe Leute, ihr definiert Euch als links. Aber da kann ich zwar eure Interessenlage sehen, aber wir müssen natürlich auch im gesamtbezirklichen Interesse gucken. Da gibt es auch noch viele gute Projekte, die das auch wollen. ${ }^{620}$ (Hervorhebung H.L.)

17 Tatsächlich fand dieser Politikansatz auch Nachahmer, die jeder emanzipatorischen Hoffnung Hohn sprechen: Im Berliner Ost-Bezirk Treptow bemühte sich Ende 2004 ein Bündnis von Nazi Straße und besuchten den Begirkshürgermeister in seiner Bürorsprechstunde (Berjiner Zeitung, 15. 7. 2004 und 30. 11. 2004)

18 Projektinterview mit Bernhard Feder, felS und Initiative für ein Soziales Zentrum, 1. Juni 2004.

19 Am 23. November 2004 verfügte der Berliner Senat auf Vorlage von Finanzsenator Thilo Sarrazin, dass leerstehende landeseigene Gebäude unter Beachtung bestimmter Auflagen für eine Zwischennutzung zu Betriebs- und Unterhaltskosten freigegeben werden können. Berïndet wurde auch diese Maßnahme rein finanzpolitisch: Der Senat erhofft sich eine Ent grond warde auch diese enatsverwaltung für Finanzen, Presseerklärung vom 23. 11. 2004: Zwischennutzung leerstehender Gebäude).

20 Projektinterview mit Cornelia Reinauer (PDS), Bezirksbürgermeisterin FriedrichshainKreuzberg, 22. September 2004.
Die Bezirksbürgermeisterin reduziert hier die sozialen Forderungen der Initiative auf die in ihnen zum Ausdruck kommenden Partikularinteressen und spricht ihnen mit Verweis auf ein nicht näher erläutertes Allgemeininteresse jede Berechtigung ab. Die implizite Prämisse dieses Arguments ist, dass die haushaltspolitischen Restriktionen, die das Land Berlin dem Bezirk auferlegt, akzeptiert werden und die daraus folgenden Konsequenzen - Sparen, Kürzen, Privatisieren - als Handlungsrahmen angenommen werden. So (und nur so) wird die Unterwerfung unter eine neoliberale Stadtpolitik zur Verwirklichung eines 'gesamtbezirklichen Interesses'. Gemäß der Devise: 'Wir müssen alle den Gürtel enger schnallen' wird die rigide Spar- und Privatisierungspolitik der Stadt gegen das Projekt eines Sozialen Zentrums in Anschlag gebracht.

Zwar ist diese Form der 'Imaginierung eines Allgemeinwohl' - bzw. der Klassencharakter des kapitalistischen Allgemeinwohls - ein generelles 'Problem' parlamentarischer Politik, wie Johannes Agnoli bereits 1968 in Die Transformation der Demokratie feststellte:

„Die politische Partei des Verfassungsstaates - dort, wo sie sich am vollsten ausgebildet hat wirkt in letzter Instanz als Klassenorgan der Konservativen, weil sie keine Klassen mehr zu kennen vorgibt, sondern nur noch 'Menschen', keine gesellschaftlich bezogene Idee, sondern nur 'Sachen'. In ihr herrschen beim Entscheidungsprozeß nicht der Drang und der Druck existenter Bevö'kerungsgruppen, sondern der 'Sachzwang'." (Agnoli 1968: 34)

Unter den Bedingungen von NPM wird diese Form der Vermachtung des politisches Feldes perfektioniert. Im Konflikt um das Soziale Zentrum etwa können sich die Bürgermeisterin Reinauer und ihre Verwaltung auf (selbstgemachte) Sachzwänge zurückziehen. Die politischen Entscheidungen der öffentlichen FunktionsträgerInnen sind längst in scheinbar unpolitische Verwaltungsund Haushaltsverfahren aufgelöst. Wer sich, wie die Initiative für ein Soziales Zentrum, gegen diese Politik sträubt, öffentliche Güter und Räume einfordert oder sie sich gar eigeninitiativ aneignet, wird als egoistischer und unsolidarischer Vertreter von Partikularinteressen diffamiert und gegen die anderen Betroffenen der Berliner Kürzungs- und Umverteilungspolitik ausgespielt.

Dabei wäre die Vergabe eines öffentlichen Raumes an die Initiative für ein Soziales Zentrum für sich genommen sicher kein unlösbares finanzpolitisches Problem. Doch der von der Initiative ausdrücklich angestrebte Dominoeffekt - andere Anti-Privatisierungsproteste sollten gestärkt und die Bezirksverwaltung dadurch unter Druck gesetzt werden - stellte eine durchaus reale Bedrohung dar. In diesem Sinne ging es in dem Konflikt um ein Soziales Zentrum (natürlich) nicht nur um eine öffentliche Liegenschaft bzw. um eine Budgetfrage, sondern auch um die politischen Kräfteverhältnisse in der Stadt.

Vor diesem Hintergrund ist es interessant, dass sich gerade progressive LokalpolitikerInnen mit der Initiative für ein Soziales Zentrum entsolidarisierten. Hier kommt das Moment der politischen Konkurrenz zwischen parlamentarischen und außerparlamentarischen Akteuren voll zum Zug. Denn eine außer- 
parlamentarische Opposition links der PDS, die durch ein Soziales Zentrum infrastrukturell gestützt worden wäre, hätte den Druck auf die parlamentarische Linke in Friedrichshain-Kreuzberg deutlich erhöht. Dies gilt um so mehr, da die PDS in Berlin auch auf Landesebene in Regierungsverantwortung stand und gemeinsam mit der SPD eine rigorose Spar- und Kürzungspolitik betrieb, die von vielen lokalen Organisationen, Einzelpersonen und Einrichtungen heftig kritisiert wurde. Die Vergabe von Räumen und Infrastruktur an Gruppen wie Attac, die Initiative Berliner Bankenskandal oder das Berliner Sozialforum hätte eine Aufwertung der außerparlamentarischen Opposition impliziert und auf lokaler Ebene den politischen Konflikt zwischen den linken Parteien verstärkt. Denn zeitgleich zu den Verhandlungen um ein Soziales Zentrum tauch te mit der Gründung der 'Wahlalternative Arbeit \& soziale Gerechtigkeit' (WASG) eine parteipolitische Konkurrenz in der politischen Arena auf, die sich in Berlin 'links' der PDS positionierte. In der Gründungsphase der Berliner WASG im Jahr 2004 war die enge Zusammenarbeit zwischen PDS und WASG bei der Bundestagswahl im September 2005 und der Zusammenschluss als 'Linkspartei' noch nicht abzusehen. Für die PDS stellte die WASG zu diesem Zeitpunkt daher eine Konkurrenz dar, deren Stärke und Zukunft noch kaum abzuschätzen war. Da in der WASG Berlin auch Einzelpersonen aus der Initiative für ein Soziales Zentrum engagiert waren, musste die lokale Stärkung der Initiative von der PDS zu diesem Zeitpunkt als Aufwertung einer Konkur renzpartei interpretiert werden.

Wo enge fiskalpolitische Entscheidungsspielräume und starke ökonomische Handlungsrationalitäten das stadtpolitische Feld abstecken, erscheinen städtische soziale Bewegungen also eher als Bedrohung und Konkurrenz denn als Verbündete der parlamentarischen Linken. Dies gilt vor allem, wenn sich die außerparlamentarischen Gruppen - wie im Fall des Konflikts um das Soziale Zentrum - nicht kooptieren und in die formalpolitische Arena integrieren lassen, sondern sich ein hohes Maß an Autonomie und Militanz bewahren (siehe auch Mayer 1998) Auch hier gilt, dass die Dynamik der politischen Konkurrenz und Exklusion nicht nur in der unternehmerischen Stadt zu beobachten ist, sondern ein grundsätzliches Merkmal parlamentarischer Demokratie darstellt. Doch unter den Bedingungen der Ökonomisierung des lokalen Staates wird diese Tendenz erheblich verschärft. So lösen sich auch die letzten lokalen Bündnisse zwischen progressiven Parlamentariern und außerparlamentarischen Linken in unliebsame Konkurrenzen auf.

\section{Schlussbemerkungen}

In den aktuellen Debatten um 'die unternehmerische Stadt' wird oftmals darauf hingewiesen, dass sich die stadtpolitische Arena in den vergangenen Jahren tendenziell für nicht-staatliche Akteure geöffnet habe. Stadtteilgruppen,
Nonprofit-Organisationen und Consulting-Firmen werden in Form von Public-Private Partnership aktiv in lokale Planungsprozesse und in die Bereitstellung sozialer Dienstleistungen eingebunden (Mayer 1994; 1999). Die hier untersuchte Berliner Fallstudie, in der sich ein tiefer Konflikt zwischen parlamentarischen und außerparlamentarischen Akteuren beobachten lässt, widerspricht dem nicht. Am Beispiel des Sozialen Zentrums wird vielmehr deutlich, dass das politische Feld, auf dem die neuen Partnerschaften geschlossen werden, eng abgesteckt ist. Die neuen Partizipationschancen gehen mit einem massiven 'realpolitischen' Anpassungsdruck auf den 'Dritten Sektor' einher. NPMTechniken spielen in diesem Zusammenhang eine wichtige Rolle, denn durch sie wird der Verhandlungsrahmen bestimmt, innerhalb dessen die Akteure der neuen Urban Governance Regime agieren. Für Stadtteilgruppen und Basisinitiativen, die sich den haushaltspolitischen 'non-decisions' nicht fügen, ist dagegen wenig Platz am 'Runden Tisch'.

In Berlin hat NPM eine besondere Not. Denn hier ist die Ökonomisierung des Städtischen zu einem erheblichen Teil das Ergebnis des sogenannten Berliner Bankenskandals, der als eine riesige Wette auf den Aufschwung-Ost, auf die Global-City Werdung Berlins und auf steigende Boden- und Immobilienpreise interpretiert werden kann. Während man auf den in den $1990 \mathrm{er}$ Jahren erhofften Berliner Boom vergeblich wartete (Krätke/Borst 2000), nutzten die politischen und ökonomischen Eliten der Stadt das Geflecht von Politik und Staatsbank (Berliner Bankgesellschaft) erfolgreich, um öffentliche Mittel in Milliardenhöhe in private Vermögen zu verwandeln (Ugarte-Chacon 2004; Ugarte-Chacón 2006). Die nach dem Rücktritt der verantwortlichen CDULandesregierung im Jahr 2001 neu gewählte SPD-PDS Koalition tat nichts, um die Konsequenzen des Bankenskandals zu mildern. Stattdessen produzierte sie einen für die verantwortlichen Akteure bislang folgenlosen parlamentarischen Untersuchungsbericht von 900 Seiten Umfang (Untersuchungsausschuss 2006). Vom Berliner Bankenskandal führt eine direkte Verbindung zur Einrichtung des sogenannten Liegenschaftsfonds, über den seit 2001 alle ungenutzten öffentlichen Immobilien privatisiert werden, sowie zu den strikten Sparauflagen des Landes und der Bezirke. Die unternehmerische Stadtpolitik Berlins, die sich in NPM-Reformen, in der Kürzung sozialer Leistungen und der Privatisierung und in-Wert-Setzung öffentlicher Räume und Güter manifestiert, erlaubt es dem Land Berlin trotz der angespannten Haushaltslage die Risikoabschirmung der Bankenfonds zu übernehmen und das groß angelegte Umverteilungsprojekt auf Jahrzehnte institutionell absichern. Vor diesem Hintergrund ist es an Zynismus kaum noch zu überbieten, wenn eine alternative Stadtpolitik 'von unten', die sich dem Berliner Sparkonzert verweigert, als illegitimes Partikularinteresse aus dem politischen Feld gedrängt wird.

Wenn die hier diskutierte Vermachtung der stadtpolitischen Arena durch NPM über den Berliner Fall hinaus verallgemeinerbar ist und als Merkmal der neo- 
liberal 'modernisierten' Stadt gelten kann, dann ergeben sich daraus schwerwiegende Konsequenzen für soziale Bewegungen und städtische Privatisierungskämpfe in der Zukunft: Die zentrale Rolle lokaler Verwaltungen im politischen Prozess gilt als herausragendes Merkmal der 'europäischen Stadt' und insbesondere des bundesdeutschen Städtewesens (siehe etwa Siebel 2004). Doch während die städtischen Verwaltungen bislang eher als 'ausgleichendes Moment' zwischen den unterschiedlichen lokalpolischen Akteuren und als eine Art 'Vertretung gesamtstädtischer Interessen' gesehen wurden, legt die Berliner Fallstudie eine andere Deutung nahe. Eine neoliberal konfigurierte Bürokratie schließt Privatisierungspolitik 'hermetisch' ab; sie gibt sich tendenziell unpolitisch, interessen- und subjektlos. Sie konstruiert einen Privatisierungszwang und vertieft die bestehenden Konkurrenzen zwischen der außerparlamentarischen Linken und progressiven Parlamentariern. So wird der neoliberale Umbau der Stadt gegen jede Form von Kritik, Protest und Diskussion immunisiert und gegen die Interventionen außerparlamentarischer Akteure und progressiver LokalpolitikerInnen abgedichtet.

\section{Literatur}

Agnoli, Johannes (1995): Der Staat des Kapitals, in: Der Staat des Kapitals und weitere Schriften zur Kritik der Politik. Agnoli, Johannes (Hg.), S. 21-89. Freiburg: ca ir

Agnoli, Johannes (1968): Die Transformation der Demokratie, in: Die Transformation der Demokratie. Agnoli, Johannes/Brückner, Peter (Hg.), S. 7-87. Frankfurt/M.

Bann Otto Suhr Institut der FU Berlin, Berlin

Bogumil, Jörg/Grohs, Stephan/ Kuhlmann, Sabine (2006): Ergebnisse und Wirkungen kommunaler Verwaltungsmodernisierung in Deutschland - Eine Evaluation nach zehn Jahren Praxiserfahrung, in: Politik und Verwaltung. PVS Sonderheft 37/2006. Bogumil, Jörg/Jann, Werner/Nullmeier, Frank (Hg.), S. 151-184

Brenner, Neil/ Heeg, Susanne (1999): Lokale Politik und Stadtentwicklung nach dem Fordismus: Möglichkeiten und Beschränkungen, in: Kurswechsel, Wien: Möglichkeiten und Grenzen einer alternativen Stadtpolitik, Nr. 2, S. 103-119

Bröckling Ulrich/ Kromentaliät der Gegenwart; Studien zur Ölonomisierung des Sozialen, Suhrkamp. Frankfurt/M.

Clarke, Susan E./ Gaile, Gary L. (1998): The Work of Cities, Minneapolis: University of Minnesota Press

felS (2004): Linksradikale Politik im Berliner Sozialforum, in: Arranca! Linke Zeitschrift, Nr. 29 , S. $21-22$

Fischer, Andreas H. (1999): Einfuihrung der Kosten- und Leistungsrechnung in der Berliner Verwaltung. Vortrag auf dem Bundeskongress 'Netzwerk Reform' am 23. Juni 1999, Berlin: Senatsverwaltung für Finanzen

Foucault, M. (1994): Überwachen und Strafen. Die Geburt des Gefängnisses, Frankfurt/M. Suhrkamp

Foucault, Michel (2000): Die Gouvernementalität, in: Gouvernementalität der Gegenwart: Studien zur Ökonomisierung des Sozialen. Bröckling, Ulrich/Krasmann, Susanne/Lemke, Thomas (Hg.), S. 41-71. Frankfurt/M.: Suhrkamp

Harvey, David (1989): From Managerialism to Entrepreneurialism: The Transformation in Urban Governance in Late Capitalism, in. Geografiska Annaler, 71 B. Jg, Nr. 1, S. 3-18

Jäggi, Max (1996): Amtlich verordnete Magersucht - New Public Management: Ein Modebegriff und seine Folgen, in: Weltwoche, Nr. 51 (Dezember 1996)
Jessop, Bob (1997): The Entrepreneurial City: Re-Imaging Localities, Redesigning Economic Governance, or Restructuring Capital?, in: Transforming Cities: Contested Governance and New Spatial Divisions. Jewson, Nick/MacGregor, Susanne (Hg.), S. 28-41. London: Routledge

Krätke, Stefan/ Borst, Renate (2000): Berlin. Metropole zwischen Boom und Krise, Opladen: Leske+Budrich

Kuhlmann, Sabine (2006): Hat das „Neue Steuerungsmodel1“ versagt? Lehren aus der Ökonomisierung von Politik und Verwaltung, in: Verwaltung und Management, Nr. 3/2006, S. 149-152

Lebuhn, Hentik (2008): Wem gehört die Stadt? Mikrokonflikte um den öffentlichen Raum in Berlin und Los Angeles, Münster: Westfalisches Dampfboot i.E.

Mayer, Margit (1994): Post-Fordist City Politics, in: Post-Fordism: A Reader. Amin, Ash (Hg.), S. 316-337. Oxford: Basil Blackwell

Mayer, Margit (1998): Städtische soziale Bewegungen, in: Neue Soziale Bewegungen - Impulse, Bilanzen und Perspektiven. Hellmann, Kai-Uwe/Klein, Ansgar/Rohde, Markus (Hg.), S. 257 271. Opladen: Westdeutscher Verlag

Mayer, Margit (1995): Urban Governance in the Post-Fordist City, in: Managing Cities. The New Urban Context. Healey, Patsy/Cameron, Stuart/Davoudi, Simin/Graham, Stephen/MadaniPour, Ali (Hg.), S. 231-249. Chichester, New York, Brisbane, Toronto, Singapore: John Wiley Pour, Ali

Mayer, Margit (1999): Urban movementes and Urban Theory in the Late-20th-Century City, in The urban moment. Cosmopolitan essays on the late 20th-Century City. Beauregard, Robert A./Body-Gendrot, Sophie (Hg.), S. 209-238. London, New Delh

Mayer, Margit (2000): Urban Social Movements in an Era of Globalization, in: Urban Movements in a Globalizing World. Hamel, Pierre/Lustiger-Thaler, Henri/Mayer, Margit (Hg.) S. 141-157. London, New York: Routledge

Senatsverwaltung (1995): Kostenrechnung in der Berliner Verwaltung (Konzeption), Berlin; Berliner Senatsverwaltung

Senatsverwaltung Berlin (2004): Einfihhrung der Kosten- und Leistungsrechnung in der Berliner Hauptverwaltung, Jahresabschlussbericht 2003 vom 7. Mai 2004.

Siebel, Walter (2004): Einleitung: Die europäische Stadt, in: Die europäische Stadt. Siebel, Walter (Hg.), S. 11-50. Frankfurt/M.: Suhrkamp

Ugarte-Chacon, Benedict (2004): Woher nehmen, wenn nicht stehlen?, Berlin, http://www.berliner-bankenskandal.de, Zugriff am 27.11. 2006: Initiative Berliner Bankenskandal

Ugarte-Chacón, Benedict (2006): Der Verkauf der Berliner Sparkasse. Kritik und Alternativen, Berlin: GUE/NGL

Untersuchungsausschuss (2006): Bericht des Untersuchungsausschusses Bankgesellschaft. Zu sammenfassung der wesentlichen Ergebnisse, Berlin: Download unter: http://www.parlamentberlin.de 